\title{
Analyse De L'impact Du Changement De La Couverture Végétale Sur La Pluie Et La Température De Surface Au Sénégal
}

\author{
Ibrahima Diba \\ Moctar Camara \\ Université Assane SECK de Ziguinchor, Sénégal
}

Doi: 10.19044/esj.2017.v13n29p270 URL:http://dx.doi.org/10.19044/esj.2017.v13n29p270

\begin{abstract}
This work aims at examining the potential impacts of vegetation change (reforestation) of the Sahel-Sahara interface on the intra-seasonal and interannual variability of the rainfall and surface temperature over Senegal using the RegCM4 model. Two runs were performed from 1990 to 2009 with a spatial resolution of $50 \mathrm{~km}\left(0.44^{\circ}\right)$ : the standard version of the RegCM4 model (control version) and the reforested one (named RegCM4_REFORESTATION). The impact of the reforestation is to decrease the surface temperature over Senegal in summer (JJAS). This decrease could be partly due to a decrease of the sensible heat flux over the southern and central Senegal and a strong increase of the latent heat flux. The reforestation also tends to increase the rainfall over the whole country and particularly in the Southwest. This rainfall increase which can also create an evaporative cooling, is consistent with the decrease of the surface temperature.
\end{abstract}

The analysis of the annual cycle over three domains of Senegal shows that the reforestation tends to strengthen the low-levels humidity of the atmosphere from January to December especially during the summer period in the North and in the center of the country. The surface temperature presents two maxima in April-May and October-November and a minimum during the summer. The reforestation has a cooling impact during the whole year (particularly in the summer) and over the center and the northern part of Senegal.

At the interannual timescale, the reforestation modifies significantly the rainfall by generally increasing it. However, there are years in which this trend is not respected and this translates into a weak correlation coefficient in the South of the country. This rainfall increase may translates into extreme hydroclimatic events such as floods. This work can be considered as a support for the Senegalese policymakers for the better planning of the 
management of adverse potential effects (such as floods, drought, heat waves, etc) of the Sahel-Sahara greening effort.

Keywords: RegCM4, Reforestation, Rainfall, Surface temperature, Senegal

\section{Résumé}

Le but de ce travail est d'examiner l'impact potentiel du changement de la couverture végétale (reforestation) de l'interface Sahel-Sahara sur la variabilité intrasaisonnière et interannuelle des précipitations et de la température de surface au Sénégal. Deux simulations ont été effectuées sur la période 1990-2009 à une résolution de $50 \mathrm{~km}$; une avec la version standard du modèle RegCM4 (ou version de contrôle) et l'autre avec celle reforestée (version RegCM4_REFORESTATION). L'impact de la reforestation se manifeste par une diminution de la température de surface sur l'ensemble du Sénégal en été (JJAS). Cette baisse serait due en partie à la diminution du flux de chaleur sensible au sud et au centre du pays et à une forte augmentation du flux de chaleur latente durant cette période. La reforestation tend à augmenter la pluie sur l'ensemble du pays et particulièrement au Sudouest. Ce renforcement de la pluie est cohérent avec la diminution de la température de surface car pouvant créer un refroidissement par évaporation. L'analyse du cycle annuel dans trois sous domaines du Sénégal montre que la reforestation tend à renforcer l'humidité des basses couches tout au long de l'année (surtout en juillet, août et septembre) au nord et au centre du pays. La température de surface présente quant à elle, présente une allure bimodale composée de deux maxima en avril-mai et octobre-novembre et d'un minimum durant l'été. La reforestation a comme impact un refroidissement de la température de surface au cours de l'année (particulièrement en été) au nord et au centre du Sénégal. A l'échelle interannuelle, la reforestation modifie significativement la pluie en l'augmentant. Cependant, il existe des années ou cette tendance n'est pas respectée et cela se traduit par un faible coefficient de corrélation au sud du pays. Cette augmentation des précipitations pourrait se traduire par une recrudescence des événements hydro-climatiques extrêmes tels que les inondations. Ce travail est donc une tentative de compréhension de l'impact potentiel de certains projets de reverdissement (comme la grande muraille verte) sur les conditions environnementales au Sénégal afin de donner aux décideurs politiques les outils nécessaires de planification pour lutter contre les éventuels effets néfastes associées (inondations, vagues de chaleur et sécheresse).

Mots-clés : RegCM4, Reforestation, Pluie, Température de surface, Sénégal 


\section{Introduction}

A l'instar des pays de l'Afrique subsaharienne, le Sénégal reste un pays vulnérable à la variabilité climatique. Dans ce pays, près de $60 \%$ de la population vit et dépend des ressources tirées de l'agriculture et de l'élevage qui sont deux activités fortement influencées par le climat. Il existe une étroite relation de dépendance entre le bon déroulement de la saison pluvieuse et les activités agricoles (Dieng et al., 2008). Sané et al., (2008) ont montré que depuis trois décennies les conditions climatiques au Sénégal sont caractérisées par une irrégularité pluviométrique et une mauvaise répartition des précipitations dans l'espace. Sagna (1988) puis Leroux (1995) ont montré que durant les trois dernières décennies, ce pays a enregistré des sécheresses plus ou moins profondes qui ont entraîné une mauvaise qualité de la saison pluvieuse entraînant par conséquent une forte dégradation des sols. Cette dégradation des sols combinée à la désertification constituent une menace pour la sécurité alimentaire et la vie des populations locales dans ce pays. Pour lutter contre cette dégradation des sols et la désertification, l'union africaine a endossé un grand projet dénommé grande muraille verte. Ce projet cherche à reboiser une vaste zone (sur une largeur de $15 \mathrm{~km}$ et une longueur de plus de $7000 \mathrm{~km}$ ) allant de Dakar (Sénégal) à Djibouti. Notre travail s'en inspire et à pour objectif d'évaluer la réponse potentielle d'un changement de la couverture végétale (reforestation) de l'interface SahelSahara sur la variabilité intra-saisonnière et interannuelle des précipitations et de la température de surface au Sénégal afin de permettre aux décideurs politiques de mettre en place des stratégies de lutte efficaces contre les éventuels effets néfastes d'un tel projet (inondations, sécheresse, etc). Ce travail est d'une importance capitale pour le Sénégal puisqu'il a été montré par Sultan, (2002) et Balme, (2004) que la variabilité pluviométrique influence directement les rendements agricoles dans ce pays dont l'agriculture est l'un des piliers de la croissance.

Une description du modèle RegCM4 ainsi que le protocole expérimental sont présentés dans la deuxième section. La troisième section analyse et discute les résultats. La conclusion est exposée dans la quatrième section.

\section{Description du modèle, des expériences, des données et méthodes Description du modèle et des expériences}

Dans cette étude, nous avons utilisé la version standard du modèle climatique régional RegCM4 développé au Centre International de Physique Théorique (ICTP-Italie-Trieste) tel que décrit dans Giorgi et al., (2012). Les processus de surface sont simulés en utilisant le modèle de surface BATS 1E (Biosphere Atmosphere Transfer Scheme) qui est dans Dickinson et al., (1993). Le modèle de Holtslag et al., (1990) est utilisé pour la 
paramétrisation de la couche limite planétaire. La pluie de grande échelle est paramétrisée grâce au schéma explicite d'humidité et de nuages (SUBEX) développé par Pal et al., 2000. Les schémas de convection utilisés sont ceux de Grell (1993) avec la fermeture de Fritsch and Chappell (1980) pour le continent et Emanuel (1991) pour l'océan. Le domaine simulé est compris entre les latitudes $10^{\circ} \mathrm{S}-47^{\circ} \mathrm{N}$ et les longitudes $27^{\circ} \mathrm{W}-47^{\circ} \mathrm{E}$ (figure 1), avec une résolution horizontale de $50 \mathrm{~km}$. Dans la direction verticale, le modèle comporte 18 niveaux. Le domaine est assez grand pour réduire les incertitudes entre les conditions aux limites latérales et le modèle. La zone ciblée est le Sénégal (figure 1). Deux expériences utilisant des types de couverture terrestre différents sont effectuées (figure 2). Ces expériences de changement de couverture végétale ont été effectuées sur une bande zonale Ouest - Est comprise entre les latitudes $15^{\circ} \mathrm{N}$ et $20^{\circ} \mathrm{N}$ (figure 2). Les noms des différents types de couverture du sol utilisés sont indiqués dans le tableau 1 (voir Diba et al., (2016) pour plus de détails sur le protocole des simulations). Dans la première expérience (figure 2a) qui est la simulation de contrôle (CTR) pour cette étude, la couverture végétale par défaut fournit par la version standard du modèle RegCM4 est utilisée. La deuxième expérience (appelée RegCM4_REFORESTATION) est identique à la première à l'exception que la couverture végétale dans la région comprise entre la bande zonale $15^{\circ} \mathrm{N}$ et $20^{\circ} \mathrm{N}$ est remplacée par une forêt indiquée par le rectangle rouge (figure 2b). La durée de simulation de chaque expérience est de 20 ans (de 1990 à 2009).

\section{Description des données et méthodes}

La simulation de contrôle (version standard du modèle RegCM4) et celle de la version modifiée (reboisée) du modèle ont été initialisées et pilotées à leurs frontières latérales par les réanalyses ERA-Interim (de résolution spatiale de $1,5^{\circ} \times 1,5^{\circ}$, Simmons et al, 2007; Uppala et al., 2008) du Centre Européen pour les Prévisions Météorologiques à Moyen Terme (CEPMMT). Les données de réanalyses sont issues d'une combinaison des résultats d'un modèle météorologique avec toutes les mesures d'observation (radiosondages, observation par satellites, avions de recherche pour des mesures, etc.) disponibles sur une période de plusieurs années. Elles sont à l'heure actuelle le meilleur moyen de décrire l'état global de l'atmosphère surtout dans les régions où les mesures sont insuffisantes : cas de l'Afrique de l'ouest. Les réanalyses ERA-Interim couvrent la période 1979 à nos jours et ont été précédemment utilisées par diverses études (Kothe and Ahrens, 2010; Oettli et al., 2011; Paeth et al.,2011 ; Camara et al., 2013 ; Diallo et al., 2013) grâce à leur bonne capacité à reproduire les différents composants du système de mousson ouest africain (Dee et al., 2011; Diallo et al., 2014). Nous avons évalué dans cette étude l'impact de la reforestation sur la pluie 
dans trois sous-domaines du Sénégal (Nord, Centre et Sud) en analysant le biais (équation 1) et l'erreur quadratique moyenne (équation 2) entre la version reforestée du modèle et celle de contrôle afin d'évaluer la capacité de la version reforestée du modèle RegCM4 à quantifier adéquatement cet impact dans ces trois sous-domaines. La méthode de Kraus (1977) pour le calcul des indices régionalisés (équation 3) est aussi utilisée pour calculer les anomalies standardisées de la pluie dans les trois sous-domaines ciblés.

$$
\begin{gathered}
\text { BIAIS }=\frac{1}{n} \sum_{i=1}^{n}\left(M R_{i}-M S_{i}\right) \\
E Q M=\sqrt{\frac{\sum_{i=1}^{n}\left(M R_{i}-M S_{i}\right)^{2}}{n}}
\end{gathered}
$$

$n:$ Pas de temps

$M R_{i}$ et $M S_{i}$ : Pluie simulée respectivement par la version reforestée du modèle RegCM4 et celle standard

$E Q M$ : Erreur quadratique moyenne

$$
I=\frac{P_{i}-\bar{P}}{\sigma}
$$

$I$ : Indice pluviométrique

$P_{i}$ : Pluviométrie de l'année i (de 1990 à 2009)

$\bar{P}$ : Pluviométrie moyenne sur la période 1990-2009 (soit 20 ans) et,

$\sigma$ : L'écart type sur cette même période 


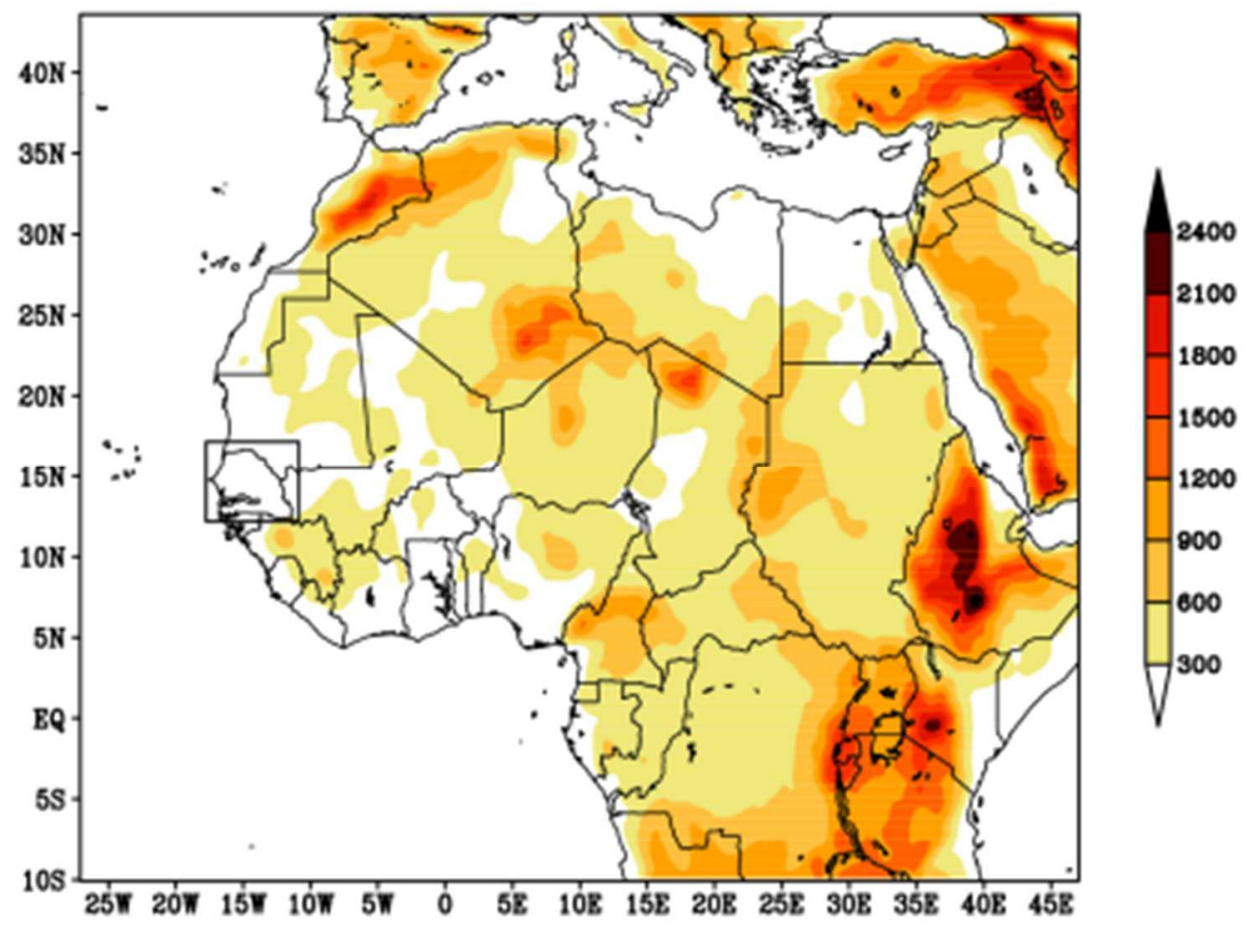

Figure 1: Topographie du domaine simulé (Afrique de l'Ouest) et la zone d'intérêt : le Sénégal en rectangle.
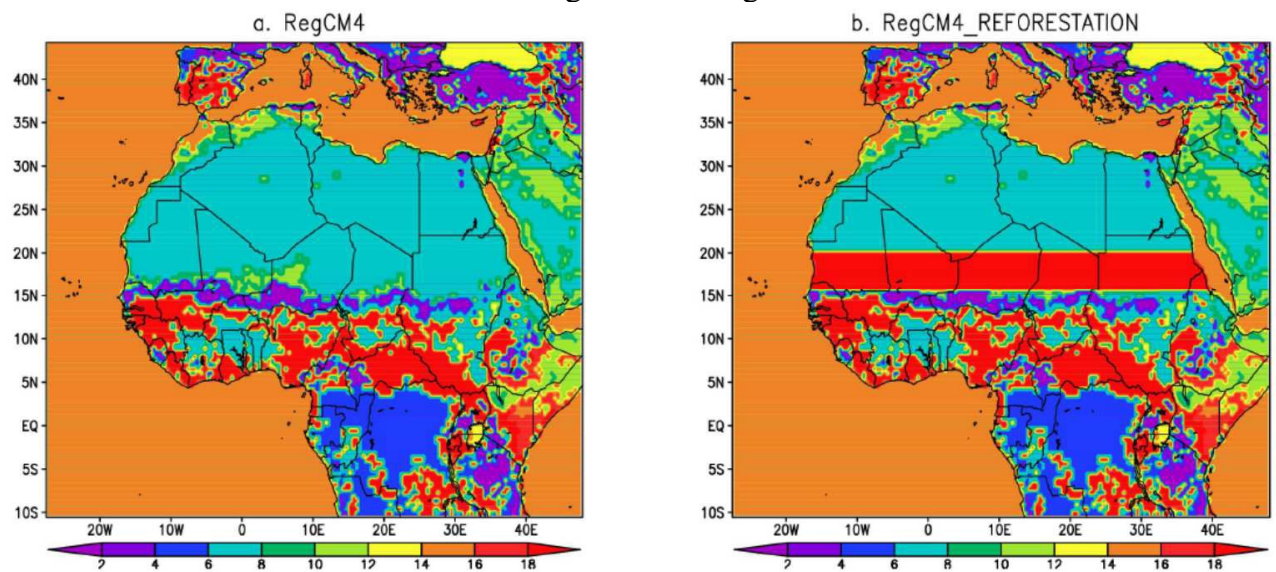

Figure 2 : Distribution des types de couverture du sol utilisés dans cet étude en (a) version standard du modèle RegCM4 et (b) version reforestée RegCM4_REFORESTATION (le rectangle en rouge indique la zone reforestée). 
Tableau 1: Types de couverture du sol utilisés dans cette étude

\begin{tabular}{|c|c|c|c|c|c|c|c|c|}
\hline$[0-2]$ & {$[2-4]$} & {$[4-6]$} & {$[6-8]$} & {$[8-10]$} & {$[10-12]$} & {$[12-14]$} & {$[14-16]$} & $\begin{array}{c}{[16-} \\
18]\end{array}$ \\
\hline Cultures & $\begin{array}{c}\text { Cultures } \\
\text { irriguées }\end{array}$ & Herbes & Désert & Semi- & $\begin{array}{c}\text { Herbes } \\
\text { hautes }\end{array}$ & $\begin{array}{c}\text { Les eaux sur } \\
\text { terre } \\
\text { (canaux, } \\
\text { lacs, } \\
\text { courtes }\end{array}$ & $\begin{array}{c}\text { Marais ou } \\
\text { tourbières }\end{array}$ & Forête \\
\hline
\end{tabular}

\section{Résultats}

Les simulations analysées dans cette étude ont été validées dans Diba et al., (2016). Les résultats ont montré que le modèle RegCM4 présente de faibles biais de pluie et de température de surface estivale traduisant ainsi la bonne capacité du modèle RegCM4 à simuler le climat de l'Afrique de l'Ouest. Ce présent travail évalue les impacts potentiels de la reforestation de l'interface Sahel-Sahara sur la pluie et la température de surface en été et aux échelles intrasaisonnière et interannuelle au Sénégal.

\section{Pluie et température de surface en été}

La figure 3 présente la distribution spatiale de la température de surface moyennée sur la période 1990-2009 pour les deux versions du modèle (reforestée et standard) et leur différence. La version standard du modèle RegCM4 (figure 3a) simule de fortes valeurs de températures excédant $30^{\circ} \mathrm{C}$ sur la partie nord-est du Sénégal durant la période estivale (Juin-Septembre; JJAS). La version reforestée du modèle reproduit assez bien ces maxima (figure 3b). Les deux versions du modèle montrent un gradient Nord-Sud avec des températures qui augmentent du Sud vers le Nord. L'impact de la reforestation (figure 3c) se manifeste par une diminution de la température sur l'ensemble du Sénégal. Cette diminution est beaucoup plus importante (jusqu'à plus de $-2.5^{\circ} \mathrm{C}$ ) sur la partie nord-est du pays. Dans le but de bien interpréter le changement sur la température de surface, les flux de chaleurs sensible et latente ont été analysés. La figure 4 présente le flux de chaleur sensible simulé par les deux versions du modèle ainsi que leur différence. La version standard du modèle RegCM4 simule de faibles valeurs de flux de chaleur sensible $\left(\leq 10 \mathrm{~W} . \mathrm{m}^{-2}\right)$ sur l'océan (figure 4a) et des valeurs inférieures à $100 \mathrm{~W} \cdot \mathrm{m}^{-2}$ au centre et au sud du pays. Par contre, les maxima de flux de chaleur sensible $\left(>100 \mathrm{~W} . \mathrm{m}^{-2}\right)$ sont obtenus au nord du pays. L'analyse de l'écart entre la version reforestée et celle standard (figure 4c) montre une diminution (augmentation) du flux de chaleur sensible au sud et au centre du Sénégal (à la frontière avec la Mauritanie). La baisse de la température sur la même zone (figure 3c) serait donc due en partie à la diminution du flux de chaleur sensible. La figure 5 présente le flux de chaleur latente pour les deux versions du modèle (reforestée et contrôle) et 
leur différence. La version de contrôle du modèle simule des valeurs de flux de chaleur latente inférieures à $50 \mathrm{~W} \cdot \mathrm{m}^{-2}$ au nord du Sénégal. Les fortes valeurs $\left(>80 \mathrm{~W} \cdot \mathrm{m}^{-2}\right)$ sont simulées au centre et au sud du pays; zones où les minima de température sont obtenues (figures $3 a, b$ ). La reforestation tend à augmenter (diminuer) le flux de chaleur latente sur l'ensemble du Sénégal (sur l'Océan Atlantique). Ce renforcement du flux de chaleur latente pourrait aussi expliquer en partie la diminution de la température de surface comme l'ont suggéré Wang et al., (2017).

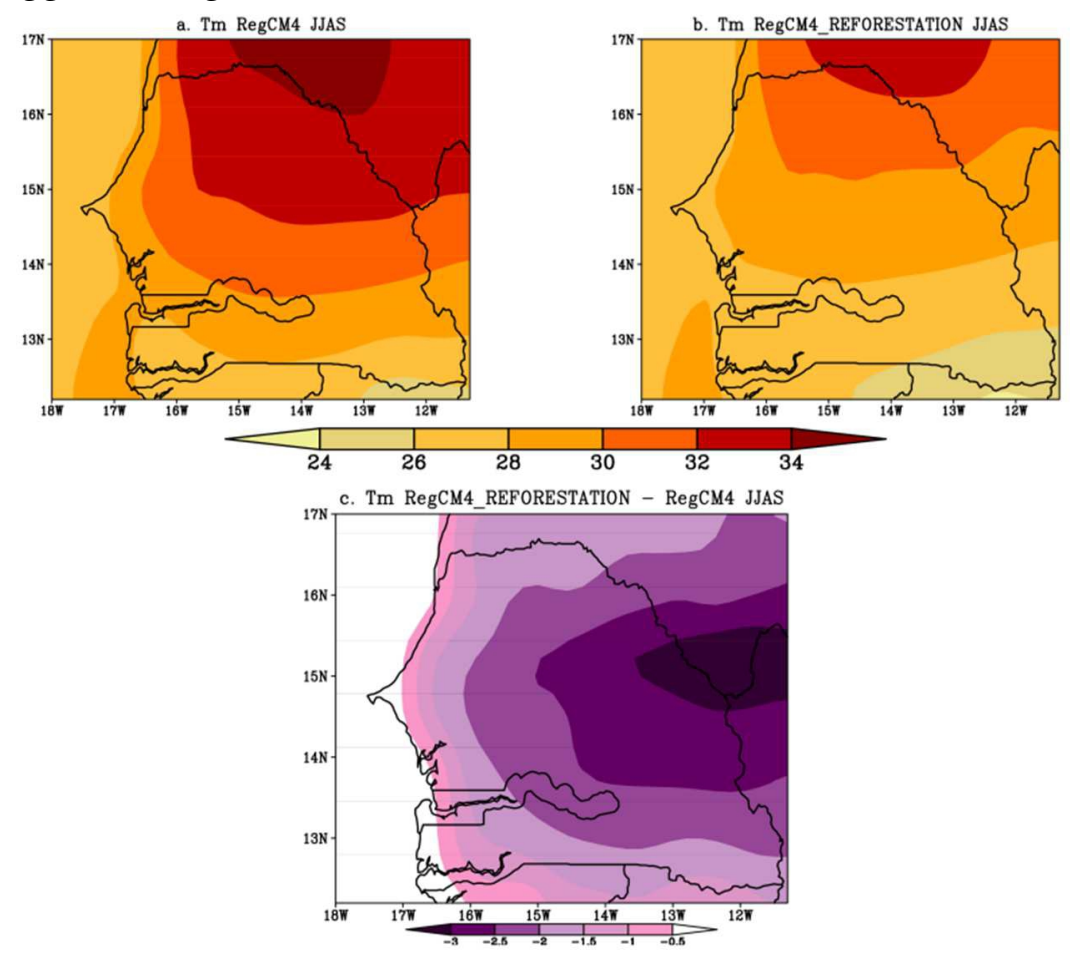

Figure 3: Température de surface $\left({ }^{\circ} \mathrm{C}\right)$ en été (JJAS) sur le Sénégal moyenné sur la période 1990-2009 pour les deux versions du modèle et leur différence. 


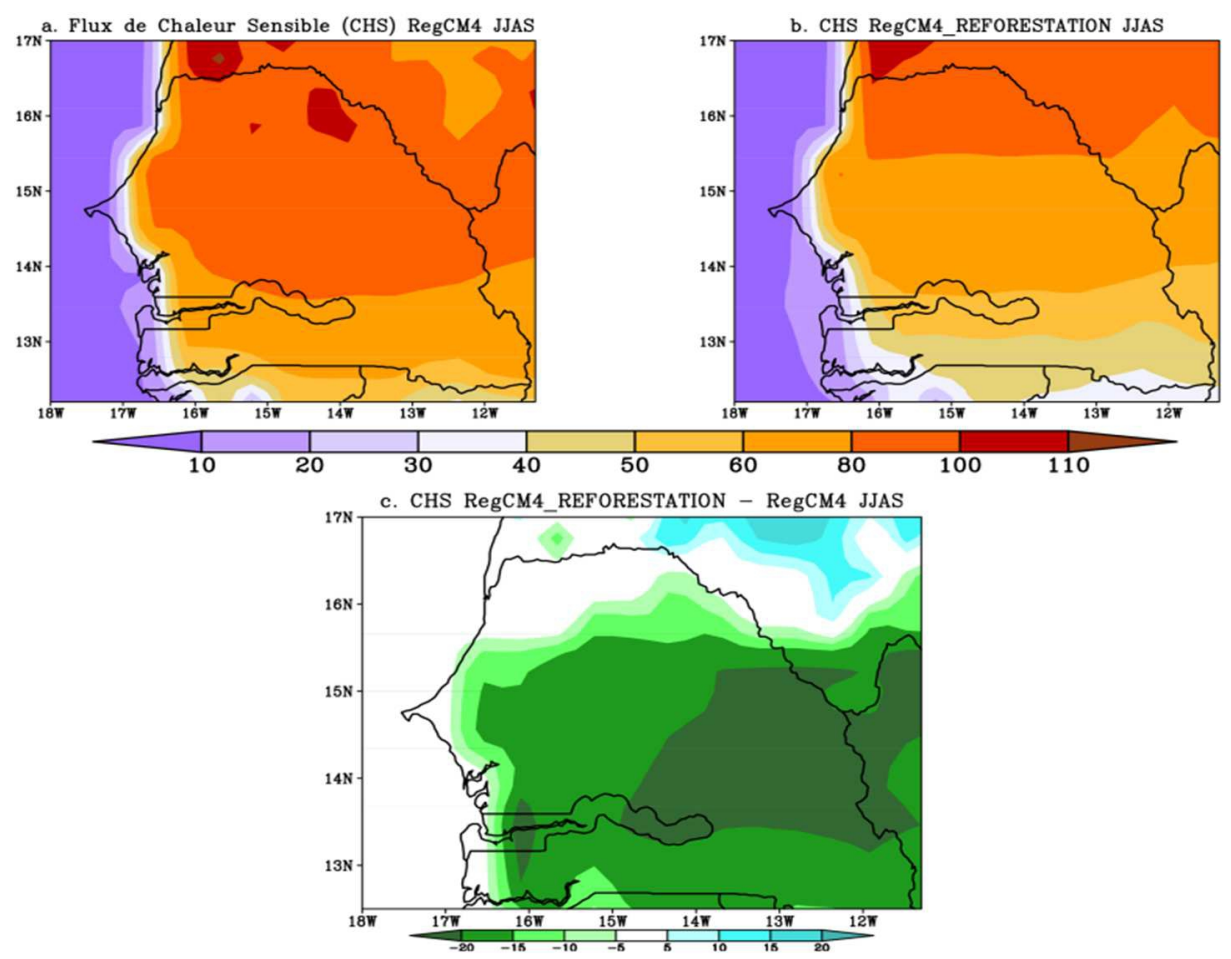

Figure 4: Flux de chaleur sensible (W.m-2) sur le Sénégal moyenné sur la période 19902009 pour les deux versions du modèle et leur différence.

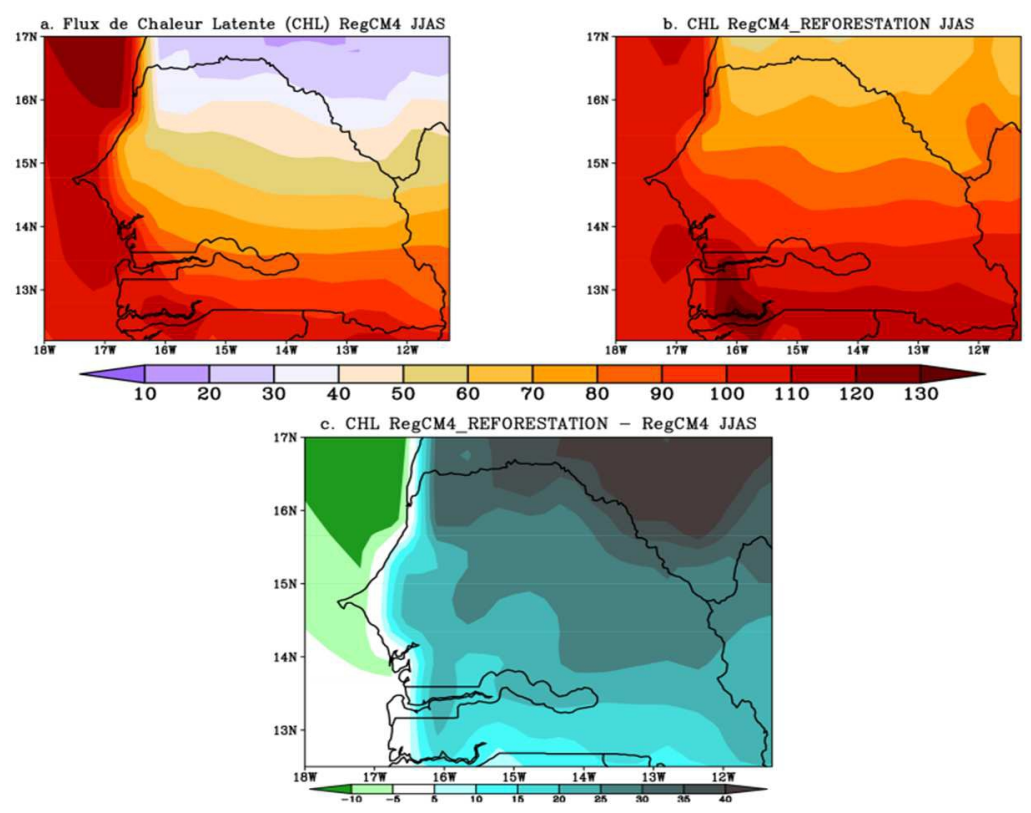

Figure 5: Flux de chaleur latente (W.m²) sur le Sénégal moyenné sur la période 1990-2009 pour les deux versions du modèle et leur différence. 
La distribution spatiale de la pluie en été (JJAS) a été analysée (figure 6). La version de contrôle du modèle simule une structure zonale de la pluie qui diminue du Sud vers le Nord (figure 6a). Cette distribution spatiale est identique à celle de la version reforestée. L'analyse de l'écart entre la version reforestée du modèle et celle de contrôle (figure 6c) montre que la reforestation augmente la pluie sur tout le Sénégal. Le maximum est enregistré au sud-ouest du pays, suivie des zones Est et Centre. Autrement dit, ce résultat montre que le reboisement affecte les précipitations aussi bien dans la zone reforestée (localement) que dans les régions lointaines. Ce résultat est cohérent avec les travaux de Diba et al., (2016), Abdou Saley et al., (2016) et Zaroug et al (2012) qui ont montré que la reforestation dans une zone peut impacter les précipitations aussi bien localement que dans les régions lointaines. Par ailleurs, ce renforcement de la pluie en JJAS peut créer un refroidissement par évaporation; ce qui est cohérent avec la diminution de la température de surface constatée durant la même période (figure 3).

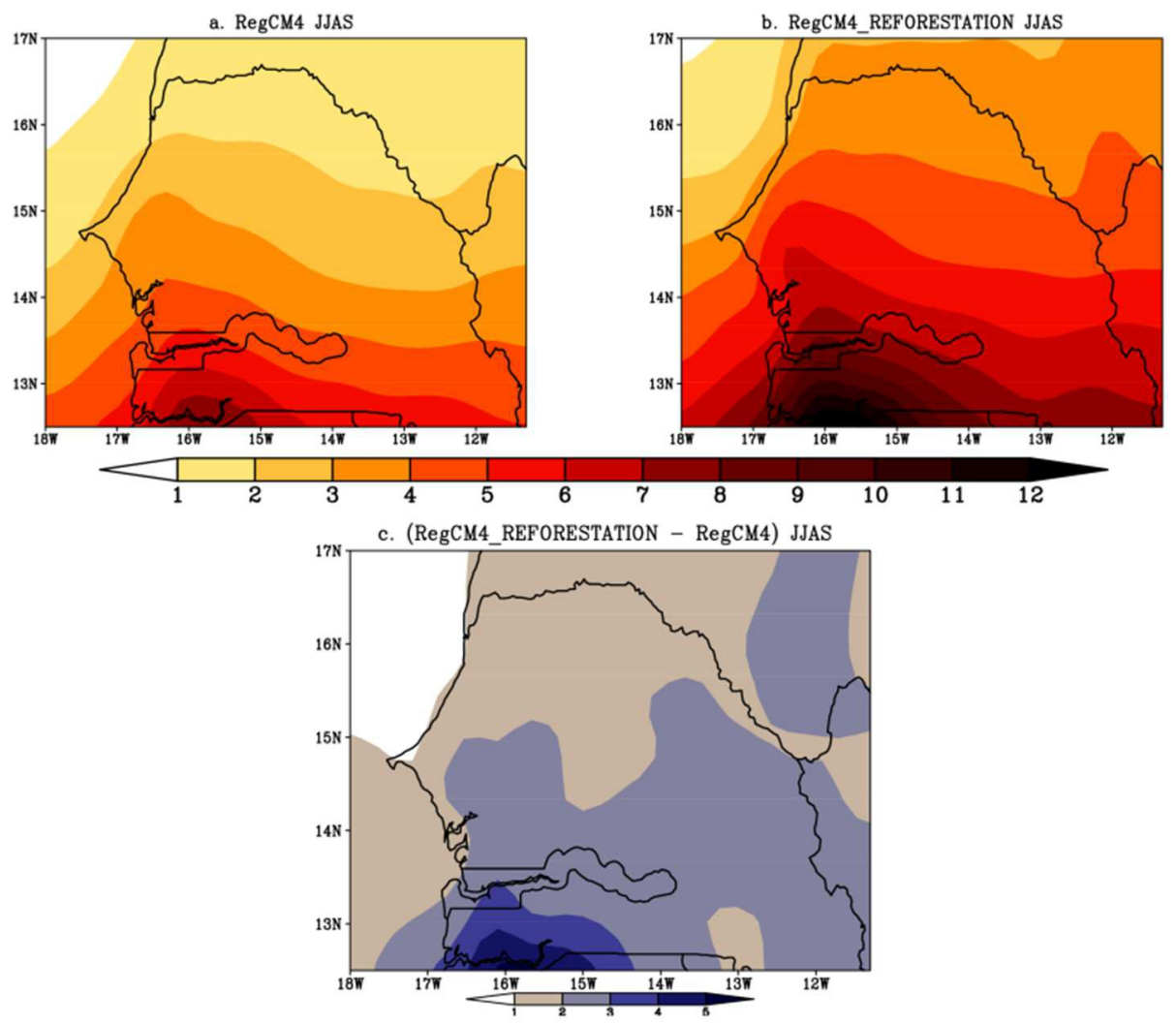

Figure 6: Pluie (mm/jr) en été (JJAS) sur le Sénégal moyennée sur la période 1990-2009:

(a) RegCM4, (b) RegCM4_REFORESTATION et (c) RegCM4_REFORESTATIONRegCM4. 


\section{Variabilité intrasaisonnière}

Dans le but de mieux diagnostiquer les impacts de la reforestation sur le climat présent du Sénégal, le cycle annuel de la pluie au nord $\left(18^{\circ} \mathrm{W}-\right.$ $\left.10^{\circ} \mathrm{W} ; 15.3^{\circ} \mathrm{N}-16.8^{\circ} \mathrm{N}\right)$, au centre $\left(18^{\circ} \mathrm{W}-10^{\circ} \mathrm{W} ; 13.7^{\circ} \mathrm{N}-15.3^{\circ} \mathrm{N}\right)$ et au sud du pays $\left(18^{\circ} \mathrm{W}-10^{\circ} \mathrm{W} ; 11.3^{\circ} \mathrm{N}-13.7^{\circ} \mathrm{N}\right)$ pour les deux versions du modèle ainsi que leur différence est présenté sur la figure 7 . La version de contrôle du modèle montre que la saison des pluies au Sénégal suit un gradient Sud-Nord avec de fortes précipitations au Sud qui s'affaiblissent en remontant vers le Nord. Le maximum de pluie est enregistré en Août dans les trois zones du pays. A partir de Septembre on observe un retrait de la bande de pluie vers le sud. La version reforestée du modèle simule bien le cycle annuel des précipitations sur le Sénégal. L'analyse de l'écart entre la version reforestée et celle standard montre que la reforestation incorporée augmente de façon générale la pluie dans les trois zones du Sénégal (Nord, Centre et Sud). Le maximum d'augmentation de précipitation est obtenu au sud du pays durant l'été (figure 7c).

La figure 8 présente le cycle annuel de la température de surface (au nord, centre et sud du Sénégal) pour les deux versions du modèle (reforestée et contrôle) et leur différence. La version standard présente une allure bimodale avec deux maxima simulés en avril-mai et octobre-novembre et un minimum détecté au mois d'août. Les faibles valeurs de températures sont simulées au sud du pays durant le mois d'Août. La version reforestée du modèle reproduit assez bien cette distribution. L'impact de la reforestation (figure 8c) se manifeste par une diminution de la température (refroidissement) sur l'ensemble du Sénégal surtout dans la période maioctobre. Ce refroidissement est beaucoup plus fort au nord et au centre du pays de Juillet à Septembre. La diminution notée lors de la période chaude (mars-avril-mai) pourrait être bénéfique aux populations locales en ce sens qu'elle pourrait se traduire par une diminution des épisodes de vagues de chaleur.

Dans le but de continuer à interpréter le changement observé sur la pluie et la température de surface, le cycle annuel de l'humidité relative (\%) intégrée dans les basses couches (entre 1000 et $850 \mathrm{hPa}$ ) est représentée sur la figure 9. La version standard du modèle présente une allure unimodale avec le maximum d'humidité relative simulé au mois d'août. Ce maximum est plus fort au sud du pays; tandis que la valeur minimale est détectée au nord du pays. La version reforestée du modèle reproduit bien cette distribution. Le cycle annuel de l'humidité relative pour les deux versions est similaire à celui de la pluie. L'analyse de l'écart entre les deux versions montre que dans les basses couches, la reforestation tend à augmenter l'humidité relative dans les trois zones considérées au Sénégal surtout dans 
la période juin-octobre. Le maximum d'augmentation est obtenu au nord et au centre du pays contrairement à la pluie qui est plus forte au sud du pays..
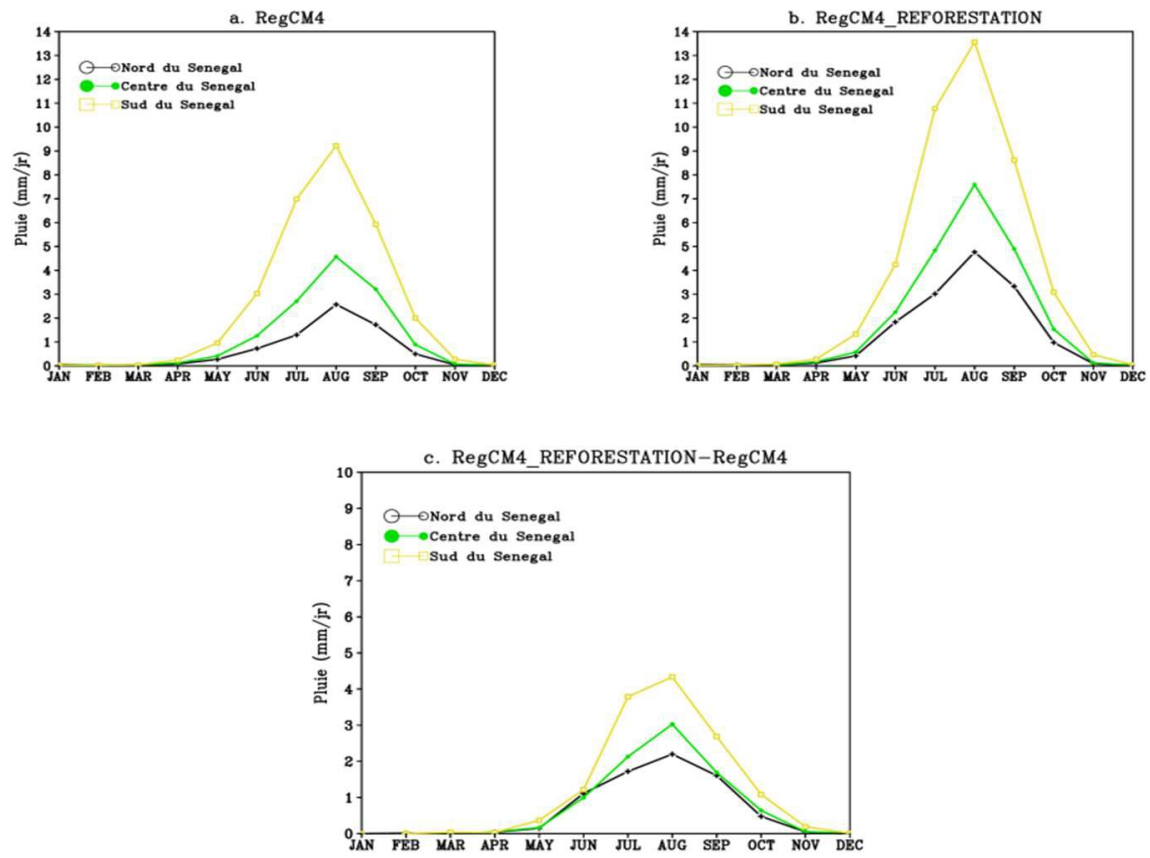

Figure 7: Cycle annuel de la pluie (mm/jr) sur le Sénégal (Nord, Centre et Sud) moyenné sur la période 1990-2009: (a) RegCM4 ; (b) RegCM4_REFORESTATIhtt ON et (c)

\section{RegCM4_REFORESTATION-RegCM4.}
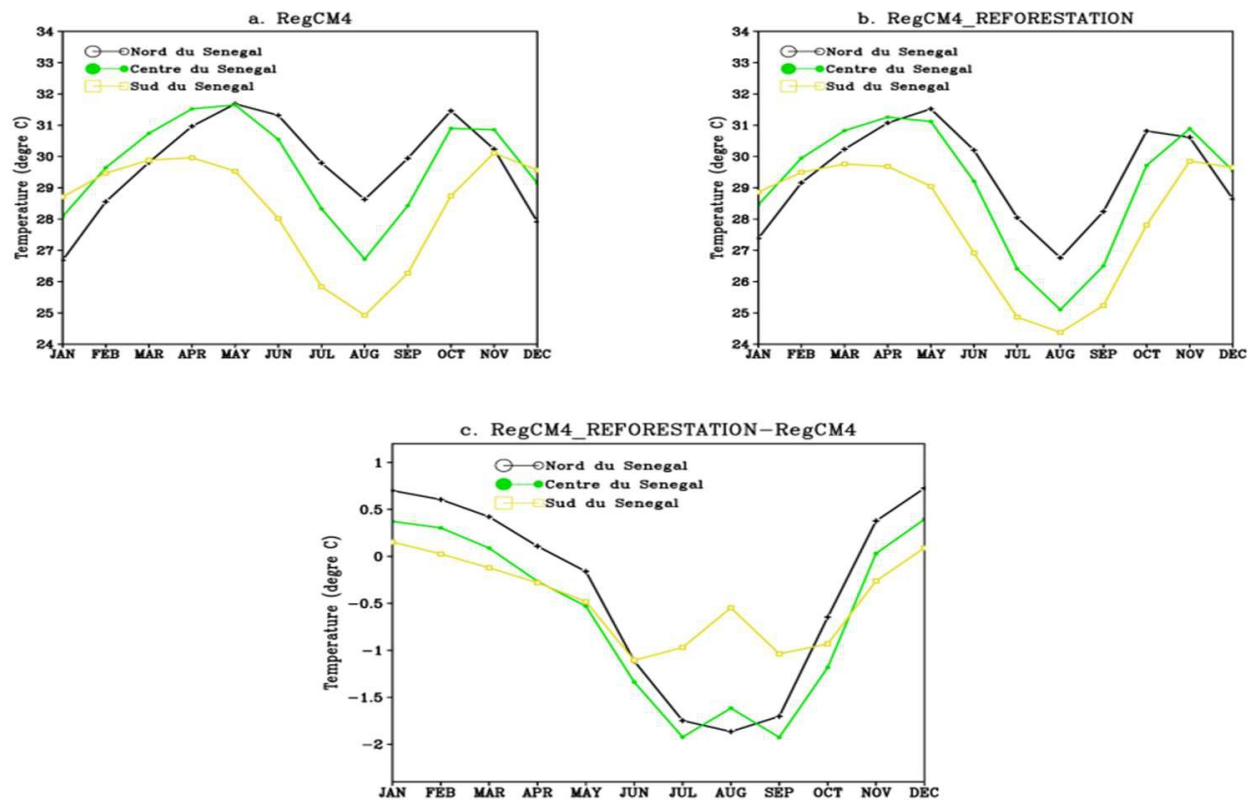

Figure 8: Cycle annuel de la température de surface $\left({ }^{\circ} \mathrm{C}\right)$ sur le Sénégal (Nord, Centre et Sud) moyenné sur la période 1990-2009: (a) RegCM4 ; (b) RegCM4_REFORESTATION et (c) RegCM4_REFORESTATION-RegCM4. 

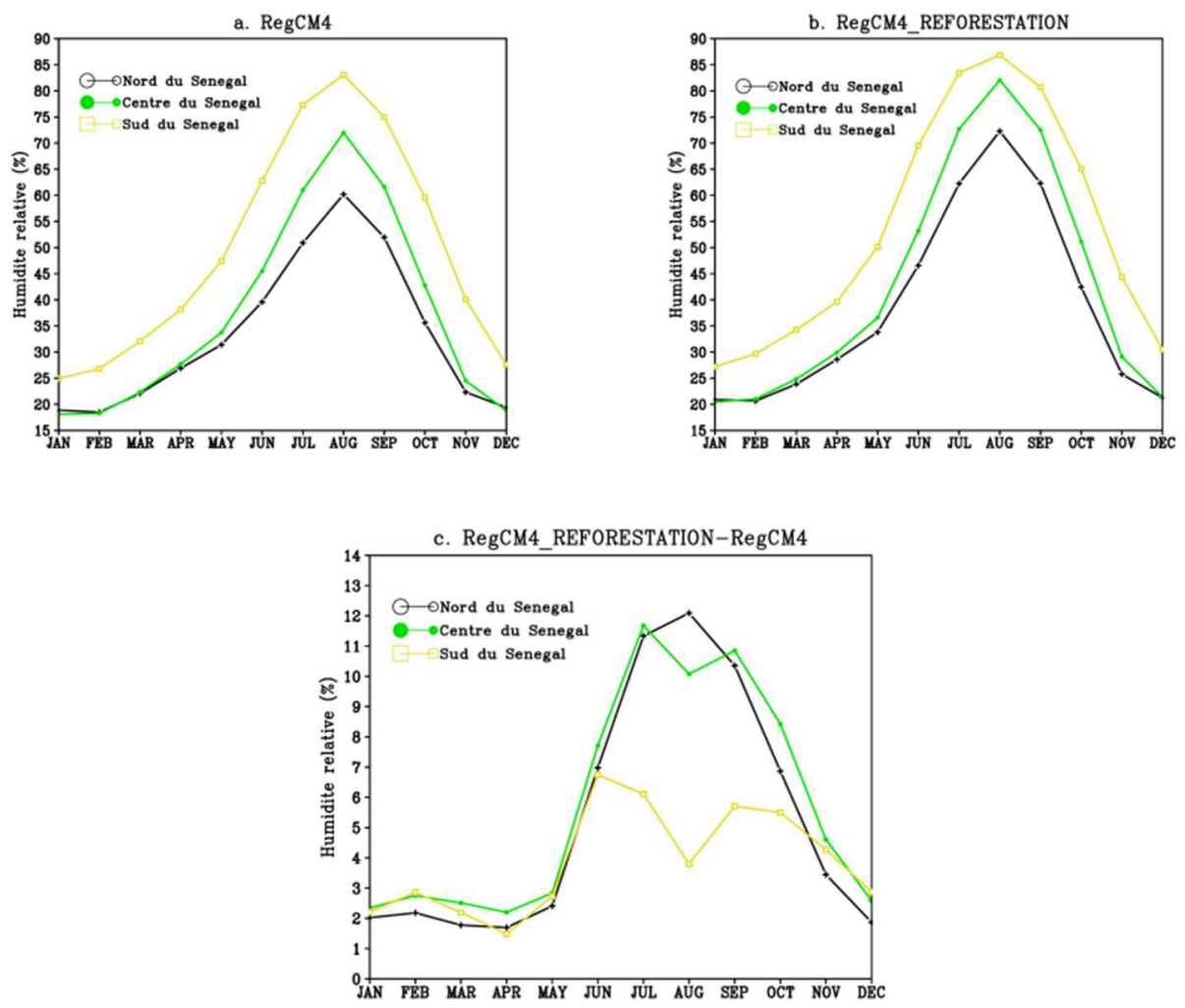

Figure 9 : Cycle annuel de l'humidité relative (\%) sur le Sénégal (Nord, Centre et Sud) moyenné sur la période 1990-2009: (a) RegCM4 ; (b) RegCM4_REFORESTATION et (c) RegCM4_REFORESTATION-RegCM4.

\section{Variabilité interannuelle}

Dans cette partie, nous avons considéré trois sous-domaines (Sud, Centre et Nord du Sénégal) afin de quantifier l'impact de la reforestation sur la pluie et la température de surface à l'échelle interannuelle. La différence entre la pluie simulée par la version reforestée et celle de contrôle du modèle (Tableau 1) montre des biais humides (valeurs positives) dans les trois zones du Sénégal. Le biais est plus humide au Sud du pays comparé au Centre et au Nord. L'impact de la reforestation (en termes d'augmentation de la pluie) est plus ressenti au sud du pays comparé au Centre et au Nord. La reforestation diminue la température dans les trois sous-domaines (Tableau 2). Cette diminution est plus ressentie au Nord du pays. Quand on considère l'erreur quadratique moyenne, elle est plus forte (plus faible) au centre du pays (Nord du pays) pour les deux paramètres (pluie et température). Ces résultats montrent que l'impact de la reforestation se traduit par une augmentation des précipitations particulièrement au Sud du pays et une baisse générale de la température de surface surtout au Nord du pays. 
Tableau 1 : Biais et erreur quadratique moyenne (EQM) de la pluie en été (JJAS) de 1990 à 2009 entre la version reforestée et celle de contrôle du modèle RegCM4 (version standard) dans les trois sous-domaines du Sénégal (Nord, Centre et Sud). L'unité est en $\mathrm{mm} / \mathrm{j}$.

\begin{tabular}{|c|c|c|}
\hline Sous-domaines & BIAIS & EQM \\
\hline $\begin{array}{c}\text { Nord du Sénégal }\left(18^{\circ} \mathrm{W}-10^{\circ} \mathrm{W} ; 15.3^{\circ} \mathrm{N}-\right. \\
\left.16.8^{\circ} \mathrm{N}\right)\end{array}$ & 1.6 & 0.4 \\
\hline $\begin{array}{c}\text { Centre du Sénégal }\left(18^{\circ} \mathrm{W}-10^{\circ} \mathrm{W} ; 13.7^{\circ} \mathrm{N}-\right. \\
\left.15.3^{\circ} \mathrm{N}\right)\end{array}$ & 2.0 & 1.4 \\
\hline $\begin{aligned} \text { Sud du Sénégal } & \left(18^{\circ} \mathrm{W}-10^{\circ} \mathrm{W} ; 11.3^{\circ} \mathrm{N}-\right. \\
& \left.13.7^{\circ} \mathrm{N}\right)\end{aligned}$ & 2.6 & 1.0 \\
\hline
\end{tabular}

Tableau 2 : Biais et erreur quadratique moyenne (EQM) de la température de surface en été (JJAS) de 1990 à 2009 entre la version reforestée et celle de contrôle du modèle RegCM4 (version standard) dans les trois sous-domaines du Sénégal (Nord, Centre et Sud). L'unité est en ${ }^{\circ} \mathrm{C}$.

\begin{tabular}{|c|c|c|}
\hline Sous-domaines & BIAIS & EQM \\
\hline Nord du Sénégal $\left(18^{\circ} \mathrm{W}-10^{\circ} \mathrm{W} ; 15.3^{\circ} \mathrm{N}-\right.$ & -0.16 & 0.65 \\
\hline $\begin{array}{c}\left.16.8^{\circ} \mathrm{N}\right) \\
\text { Centre du Sénégal }\left(18^{\circ} \mathrm{W}-10^{\circ} \mathrm{W} ; 13.7^{\circ} \mathrm{N}-\right. \\
\left.15.3^{\circ} \mathrm{N}\right)\end{array}$ & -0.11 & 0.71 \\
\hline $\begin{array}{c}\left(18^{\circ} \mathrm{W}-10^{\circ} \mathrm{W} ; 11.3^{\circ} \mathrm{N}-\right. \\
\left.13.7^{\circ} \mathrm{N}\right)\end{array}$ & -0.08 & \\
\hline
\end{tabular}

La figure 10 présente les anomalies standardisées de pluie en été (JJAS) dans les trois sous-domaines considérés au Sénégal sur la période 1990-2009 pour les deux versions du modèle. La version standard du modèle simule une forte variabilité interannuelle de la pluviométrie de 1990 à 2009. Cette période est caractérisée par une alternance brutale entre années très humides et années très sèches dans les trois sous domaines du Sénégal même si on peut considérer trois (3) périodes distinctes : la pluie diminue durant la période 1990-1995, pour ensuite connaître une tendance à la hausse jusqu'en 2005 et finir par décroître entre 2006 et 2009.-La version reforestée reproduit bien cette variabilité interannuelle des précipitations. Le coefficient de corrélation entre la version reforestée du modèle et celle de contrôle a été calculé et représenté sur la figure 10. Une corrélation positive dépassant 0.6 est obtenue au Centre du Sénégal. Néanmoins la corrélation reste faible (0.42) au Sud du pays et assez élevée (0.64) au centre du pays. Les deux versions présentent généralement les mêmes signes d'anomalie même si l'intensité diffère. Cependant, il existe des années durant lesquelles les sens des anomalies diffèrent et cela se répercute sur le coefficient de corrélation qui reste faible dans la zone sud (0.42).

La figure 11 présente les anomalies standardisées de la température de surface en été dans les trois sous-domaines considérés au Sénégal sur la période 1990-2009 pour les deux versions du modèle. La version standard du modèle présente trois phases: un réchauffement dans la période 1990-1999, 
suivi d'un refoidissement entre 2000 et 2005. La période 2006-2009 correspond à une augementation de la température de surface. La version reforestée montre une tendance similaire.

Les deux versions du modèle présentent généralement les mêmes signes d'anomalie même si l'intensité diffère et cela se répercute sur le coefficient de corrélation qui reste élevé $(\geq 0.90)$ dans les trois sous domaines du Sénégal.

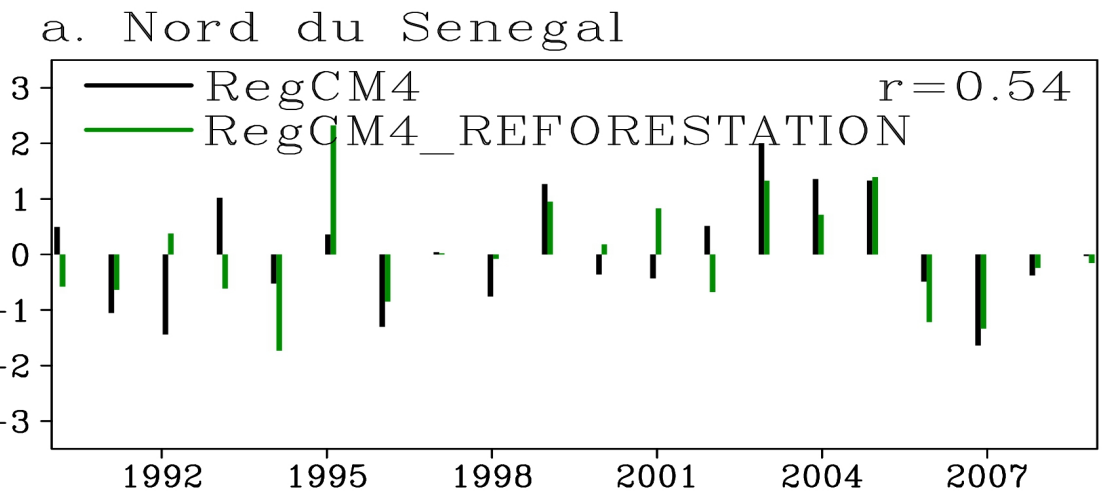

b. Centre du Senegal
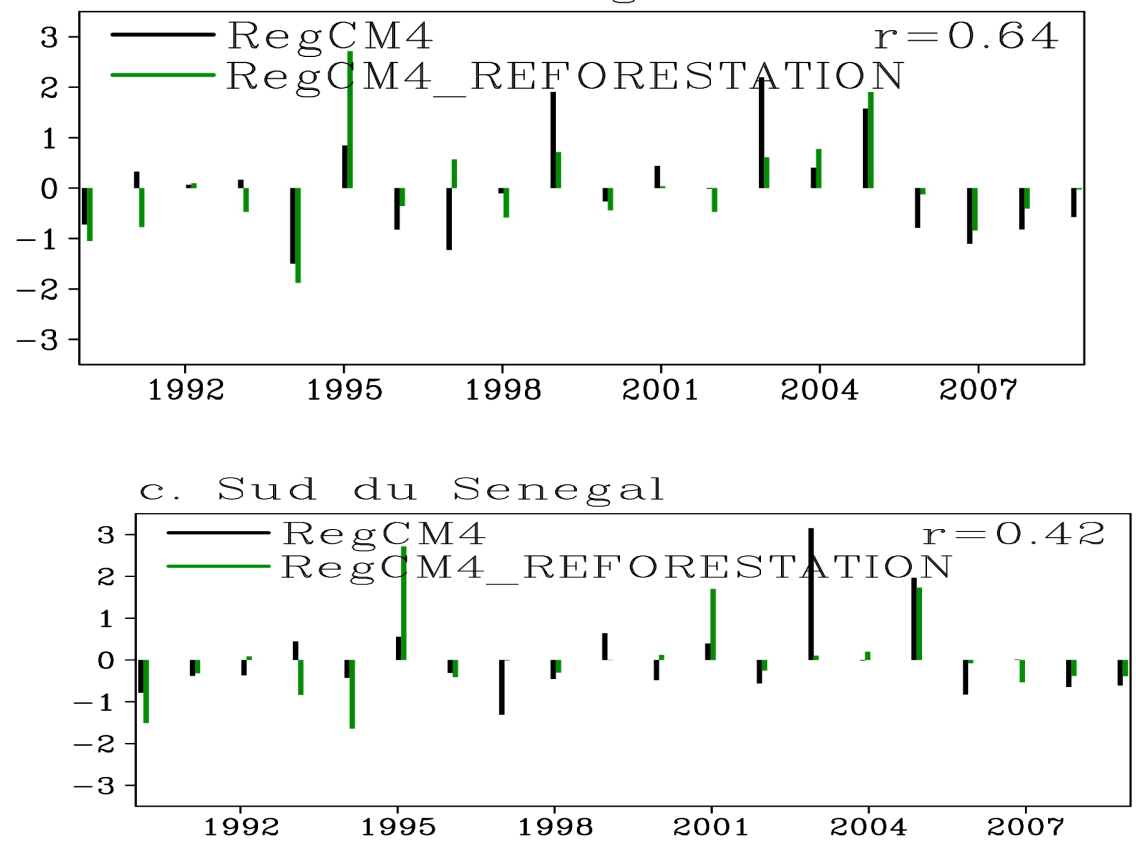

Figure 10 : Variabilité interannuelle de l'anomalie standardisée de la pluviométrie pendant la saison des pluies (Juin-Juillet-Août-Septembre) pour RegCM4 (noire) et RegCM4_REFORESTATION (vert) : (a) au Nord du Sénégal, (b) au Centre du Sénégal et (c) au Sud du Sénégal. 
a. Nord du Senegal

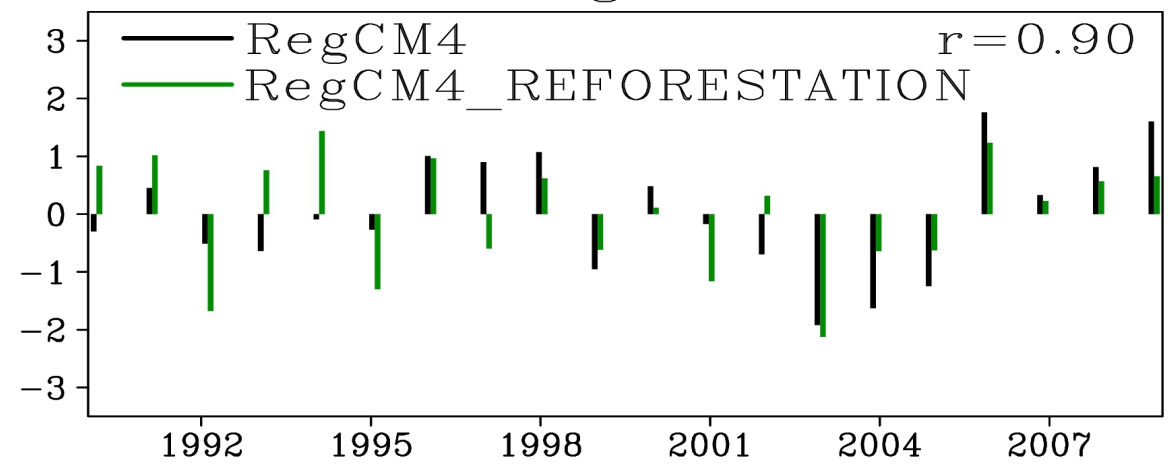

b. Centre du Senegal

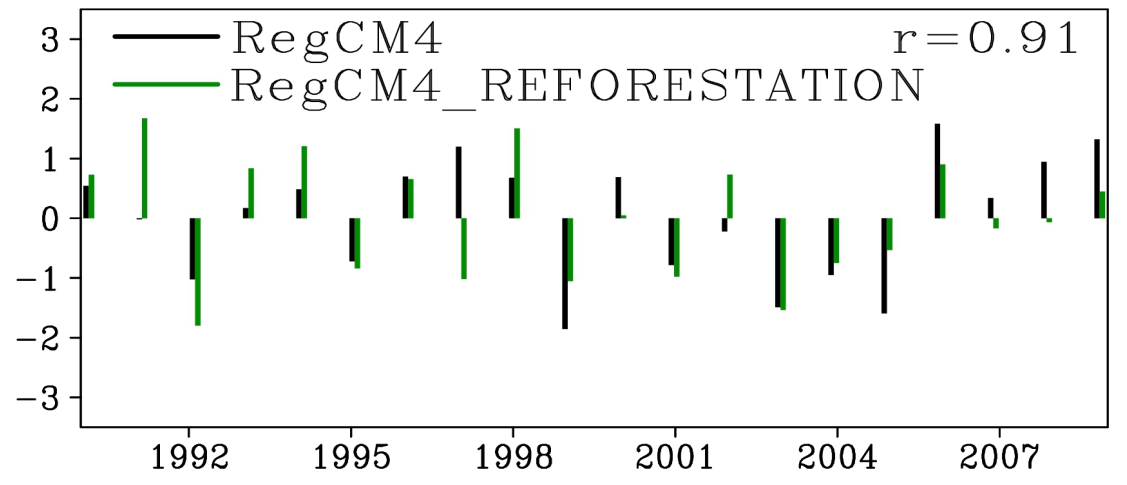

c. Sud du Senegal

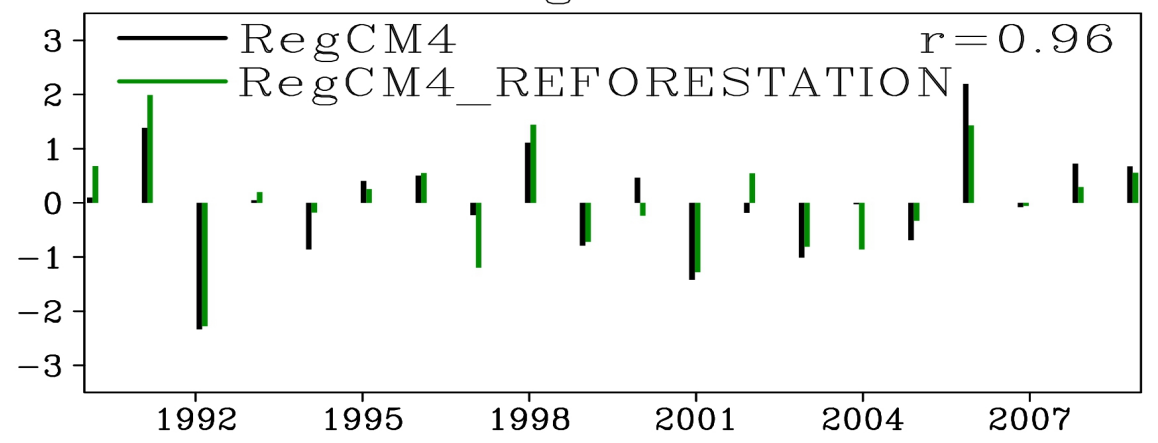

Figure 11 : Variabilité interannuelle de l'anomalie standardisée de la température de surface pendant la saison des pluies (Juin-Juillet-Août-Septembre) pour RegCM4 (noire) et RegCM4_REFORESTATION (vert) : (a) au Nord du Sénégal, (b) au Centre du Sénégal et (c) au Sud du Sénégal. 


\section{Conclusion}

Dans cette étude, les simulations effectuées à une résolution de $50 \mathrm{~km}$ avec la version standard du modèle RegCM4 et celle reforestée nommée RegCM4_REFORESTATION ont été analysées afin d'isoler les impacts potentiels de la reforestation de l'interface Sahel-Sahara sur la pluie et la température de surface au Sénégal durant l'été et aux échelles intrasaisonnière et interannuelle. Ces simulations sont toutes forcées par la dernière version de réanalyses ERA-Interim du Centre Européen pour les Prévisions Météorologiques à Moyen Terme (CEPMMT). La version de contrôle du modèle montre de fortes valeurs de températures excédant $30^{\circ} \mathrm{C}$ durant la saison pluvieuse (Juin-Septembre; JJAS). L'impact de la reforestation se manifeste par une diminution de la température sur l'ensemble du Sénégal. Cette baisse serait due en partie à la diminution du flux de chaleur sensible et au renforcement du flux de chaleur latente. Quand on considère la pluie, les résultats montrent que la saison des pluies au Sénégal suit un gradient Sud-Nord avec de fortes précipitations au Sud qui s'affaiblissent en remontant vers le Nord. L'impact de la reforestation se traduit par un renforcement de la pluie sur l'ensemble du Sénégal et plus particulièrement au Sud-ouest.

L'analyse de la variabilité intra-saisonnière montre que l'impact de la reforestation (en termes d'augmentation de la pluie) est plus ressenti au sud du pays durant les mois de juillet et Août. A l'échelle interannuelle, la reforestation modifie significativement la pluie en l'augmentant sur l'ensemble du pays particulièrement au Sud et au Centre du pays. Cependant, il existe des années durant lesquelles la reforestation n'augmente pas la pluie et cela se traduit par un faible coefficient de corrélation au Sud du pays. Ces résultats suggèrent que ce changement de couverture végétale peut avoir un impact positif sur l'agriculture au Sénégal qui est un des secteurs privilégiés pour stimuler la croissance et améliorer la sécurité alimentaire. Cependant, une forte augmentation des quantités de pluie peut engendrer des conséquences néfastes telles que les inondations à l'image de celles connues par le pays (Sénégal) en 2012. Malgré les résultats obtenus dans ce travail, d'autres études complémentaires sont nécessaires pour mieux diagnostiquer et expliciter les impacts de la reforestation sur la variabilité intra-saisonnière et interannuelle des précipitations et de la température de surface au Sénégal pour le climat présent et futur en utilisant des scenarios de changements climatiques.

\section{References :}

1. Dieng O., R. Pascal and S. Louvet, 2008: Variabilité intrasaisonnière des précipitations au Sénégal (1951-1996). Sécheresse; 19 (2): 87-93 
2. Sané T., Diop M., and P. Sagna, 2008 : Étude de la qualité de la saison pluvieuse en Haute-Casamance (Sud Sénégal). Sécheresse ; 19 (1) : 23-8

3. Sagna P, 1988: Étude des lignes de grains en Afrique de l'Ouest. Thèse de doctorat de 3 e cycle de géographie. Dakar, Ucad.

4. Leroux M, 1995: La dynamique de la Grande sécheresse sahélienne. Rev Geogr Lyon ; 70 :223-32.

5. Sultan B., 2002 : Étude de la mise en place de la mousson en Afrique de l'Ouest et de la variabilité intra-saisonnière de la convection. Applications à la sensibilité des rendements agricoles. Thèse de doctorat, Université de Paris VII.

6. Balme M., 2004 : Analyse du régime pluviométrique sahélien dans une perspective hydrologique et agronomique. Etude de l'impact de sa variabilité sur la culture du mil. Thèse de doctorat, Institut national polytechnique de Grenoble.

7. Giorgi F., E. Coppola, F. Solmon, L. Mariotti, M.B. Sylla, X. Bi, N. Elguindi, GT. Diro, V. Nair, G. Giuliani, S. Cozzini, I. Guettler, T. O'Brien, A. Tawfik, A. Shalaby, AS. Zakey, A. Steiner, F. Stordal, L. Sloan, C. Brankovic, 2012: RegCM4: Model description and preliminary tests over multiple CORDEX domains. Climate Research 52: 7-29. DOI: 10.3354/cr01018.

8. Dickinson R. E., Henderson S. A., and P. J. Kennedy, 1993: Biosphere-Atmosphere Transfer Scheme (BATS) version 1E as coupled to the NCAR Community Climate Model. NCAR Tech. rep. $T N-387+S T R, 72$ pp.

9. Holtslag A.A.M., Bruijn de E.I.F and H.L. Pan, 1990: A high resolution air mass transformation model for short-range weather forecasting. Mon. Wea. Rev., 118, 1561-1575

10. Pal, J. S., Small, E. and EAB. Eltahir, 2000: Simulation of regional-scale water and energy budgets: representation of subgrid cloud and precipitation processes within RegCM. Journal of Geophysical Research, 105, 29579-29594.

11. Grell, G. A, 1993: Prognostic evaluation of assumptions used by cumulus parameterizations. Monthly Weather Review, 121, 764-787

12. Fritsch, J. M, and C. F. Chappell, 1980: Numerical prediction of convectively driven mesoscale pressure systems. Part I: Convective parameterization. Journal of the Atmospheric Sciences, 37, 17221733.

13. Emanuel K.A., 1991: A scheme for representing cumulus convection in large-scale models. J. Atmos. Sci., 48, 2313-2335.

14. Diba I., Camara M. and A.B Sarr, 2016: Impacts of the SahelSahara Interface Reforestation on West African Climate: Intra- 
seasonal Variability and Extreme Precipitation Events. Advances in Meteorology, vol. 2016, Article ID 3262451, 20 pages.

15. Simmons AS., D. Uppala and S. Kobayashi, 2007: ERA-Interim: new ECMWF reanalysis products from 1989 onwards. $\boldsymbol{E C M W F}$ Newsletter 110: 29-35.

16. Uppala S., D. Dee, S. Kobayashi, P. Berrisford and A. Simmons, 2008: Towards a climate data assimilation system: status update of ERA-Interim. ECMWF Newsletter 115: 12-8.

17. Kothe S. and B. Ahrens, 2010: On the radiation budget in regional climate simulations for West Africa. Journal of Geophysical Research 115: D23120. doi: 10.1029/2010JD014331.

18. Oettli P., B. Sultan, C. Baron and M. Vrac, 2011: Are regional climate models relevant for crop yield prediction in West Africa? Environmental Research Letter 6: 014008. doi: 10.1088/17489326/6/1/014008.

19. Paeth H., N. M. Hall, M. A. Gaertner et al., 2011. Progress in regional downscaling of West African precipitation. Atmospheric Science Letters, vol. 12, no. 1, pp. 75-82.

20. Camara M., Diedhiou A., Sow B.A., Diallo M.D., Diatta S., Mbaye I., Diallo I., 2013 : Analyse de la pluie simulée par les modèles climatiques régionaux de CORDEX en Afrique de l'Ouest. Sécheresse 24: 14-28. doi: 10.1684/sec.2013.0375.

21. Dee and co-authors, 2011: The ERA-Interim reanalysis: configuration and performance of the data assimilation system. Quarterly Journal of the Royal Meteorology Society 137: 553-597. doi:10.1002/qj. 828

22. Diallo I., Sylla M.B., Camara M. and A.T. Gaye, 2013: Interannual variability of rainfall over the Sahel based on multiple regional climate models simulations. Theoretical and Applied Climatology 113 (1-2): 351-362. DOI: 10.1007/s00704-012-0791.

23. Diallo I., Bain C.L., Gaye A.T., Moufouma-Okia W., Niang C., Dieng M.D.B and R Graham, 2014: Simulation of the West African monsoon onset using the HadGEM3-RA regional climate model. Clim Dyn 43:575-594. DOI 10.1007/s00382-014-2219-0.

24. Kraus EB, 1977: Subtropical droughts and cross equatorial energy transports. Monthly Weather Review 105: 1009-18.

25. Wang L. and Kevin K. W. Cheung, 2017 : Potential impact of reforestation programmes and uncertainties in land cover effects over the loess plateau: a regional climate modeling study. Climatic Change. DOI 10.1007/s10584-016-1848-1.

26. Abdou Saley I. A., Sanda S. I., Bell J. P., Ly M., Salack S., Fode M., and A. Diedhiou, 2016 : Impacts potentiels de la Grande 
Muraille Verte sur le climat du Sahel et de l'Afrique de l'Ouest, Annales de l'Université Abdou Moumouni, Volume 1, Tome XXI-A. 27. Zaroug MAH, Sylla MB, Giorgi F, Eltahir EAB, Aggarwal PK, 2013: A sensitivity study on the role of the Swamps of Southern Sudan in the summer climate of North Africa using a regional climate model. Theoret Appl Climatol, Theor Appl Climatol, 113:63-81 DOI 10.1007/s00704-012-0751-6 